\section{Resistance to Twospotted Spider Mite and Strawberry Aphid in Fragaria chiloensis, F. virginiana, and $F$. Xananassa Clones}

\author{
Carl H. Shanks, Jr. \\ Washington State University, Research and Extension Unit, 1919 North East \\ 78th Street, Vancouver, WA 98665-9752
}

Patrick P. Moore

Washington State University, Research and Extension Center, 7612 Pioneer Way East, Puyallup, WA 98371-4998

\begin{abstract}
Additional index words. strawberry, Fragaria $\times$ ananassa, Fragaria chiloensis, Fragaria virginiana, Tetranychus urticae, Chaetosiphon fragaefolii
\end{abstract}

Abstract. More than 170 clones of Fragaria $\times$ ananassa Duch, $F$. chiloensis (L.) Duch. $F$. virginiana Duch. were tested for resistance to the twospotted spider mite (Tetranychus urticae Koch). Twenty-seven clones had $>75 \%$ fewer mites than did $F$. xananassa 'Totem', a susceptible clone. About two-thirds of the clones also were tested for resistance to the strawberry aphid [Chaetosiphon fragaefolii (Cockerell)]. Survival and reproduction was significantly lower on two clones each of $F$. ×ananassa and $F$. virginiana than on 'Totem'.

The twospotted spider mite (Tetranychus urticae) is a common pest of cultivated strawberries (Fragaria $\times$ ananassa). The strawberry aphid (Chaetosiphon fragaefolii) transmits several strawberry viruses. Fragaria clones vary considerably in their resistance to these pests, and these traits have been researched by several groups (Hancock et al., 1991). Giménez-Ferrer et al. (1993) reported further in vitro screening for mite resistance in strawberry cultivars. In greenhouse tests, Shanks and Garth (1992) reported on the resistance of plants of several Fragaria spp. clones to the strawberry aphid. They found that the strawberry aphid survived $<5$ days and reproduction ceased on aphid-resistant clones of $F$. chiloensis from California, Oregon, and Washington. Our paper reports the relative susceptibility of a large collection of $F$. ×ananassa, $F$. chiloensis from Chile, and $F$. virginiana clones to these pests in a greenhouse trial.

\section{Materials and Methods}

Thirty-nine cultivars and selections of $F$. $\times$ ananassa [from the National Clonal Germplasm Repository (NCGR), Corvallis, Ore.; Sakuma Bros. Farms, Burlington, Wash.; and

Received for publication 6 Sept. 1994. Accepted for publication 17 Jan. 1995. Research conducted under Project nos. 1957 and 0038, Agricultural Research Center, College of Agriculture and Home Economics, Pullman, Wash. Partial support was provided by a grant from the U.S. Dept. of Agriculture. We thank Sakuma Bros. Farms, Burlington, Wash., for Fragaria Xananassa plants. Fragaria virginiana plants were supplied by Kim Hummer (FRA series) and Margaret Stahler (M.S. series). Thanks are due to Jeanette Bergen for technical assistance. The cost of publishing this paper was defrayed in part by the payment of page charges. Under postal regulations, this paper therefore must be hereby marked advertisement solely to indicate this fact. various breeders]; 32 clones of $F$. chiloensis collected in Chile in 1990 (Cameron et al., 1991); 20 clones of $F$. virginiana from Kentucky (11), New Hampshire (3), Oregon (2), Pennsylvania (1), Vermont (1), and Wyoming (2) (K. Hummer, NCGR ); and 13 clones of $F$.

Table 1. Total number of twospotted spider mites on Fragaria clones from 20 April to 29 June 1993 in a greenhouse trial, Vancouver, Wash

\begin{tabular}{|c|c|c|c|c|c|}
\hline Clone & Species $^{2}$ & $\begin{array}{l}\text { No. mites/three } \\
\text { leaflets } \pm \mathrm{SE}\end{array}$ & Clone & Species $^{2}$ & $\begin{array}{c}\text { No. mites/three } \\
\text { leaflets } \pm \mathrm{SE}\end{array}$ \\
\hline Totem & $\mathrm{F} \times \mathrm{a}$ & $408 \pm 187$ & $342-\mathrm{A}-65$ & $\mathrm{~F} \times \mathrm{a}$ & $226 \pm 67$ \\
\hline WSU 88061-5 & $\mathrm{B} \times \mathrm{Fc}$ & $37 \pm 13$ & TDT 1D & $\mathrm{Fc}$ & $228 \pm 31$ \\
\hline PNN 6A & $\mathrm{Fc}$ & $55 \pm 20$ & WSU 88061-2 & $\mathrm{B} \times \mathrm{Fc}$ & $231 \pm 58$ \\
\hline YEN 1H & $\mathrm{Fc}$ & $55 \pm 18$ & PUR 1A-2 & $\mathrm{Fc}$ & $240 \pm 41$ \\
\hline LCO 1C & $\mathrm{Fc}$ & $56.3 \pm 18$ & FRA 1180 & $\mathrm{Fv}$ & $240 \pm 52$ \\
\hline WSU 88061-4 & $\mathrm{B} \times \mathrm{Fc}$ & $76 \pm 9$ & Gorella & $\mathrm{F} \times \mathrm{a}$ & $254 \pm 67$ \\
\hline TDT 5B & $\mathrm{Fc}$ & $85 \pm 16$ & M.S. 6-4 & $\mathrm{Fv}$ & $256 \pm 57$ \\
\hline FRA 472 & $\mathrm{Fv}$ & 90 (2 plants) & FRA 993 & $\mathrm{Fv}$ & $263 \pm 35$ \\
\hline Cavendish & $\mathrm{F} \times \mathrm{a}$ & $102 \pm 38$ & Earliglow & $\mathrm{F} \times \mathrm{a}$ & $267 \pm 78$ \\
\hline YEN 1I & $\mathrm{Fc}$ & $114 \pm 28$ & FRA 1170 & $\mathrm{Fv}$ & $276 \pm 67$ \\
\hline MAU 1C & $\mathrm{Fc}$ & $115 \pm 47$ & FRA 960 & Fv & $279 \pm 43$ \\
\hline WSU 88061-6 & $\mathrm{B} \times \mathrm{Fc}$ & $118 \pm 45$ & Pajaro & $\mathrm{F} \times \mathrm{a}$ & $282 \pm 53$ \\
\hline BAM 1E & $\mathrm{Fc}$ & $119 \pm 39$ & WSU 2068 & $\mathrm{~F} \times \mathrm{a}$ & $284 \pm 67$ \\
\hline YEN 1Q & $\mathrm{Fc}$ & $119 \pm 33$ & FRA 1007 & $\mathrm{Fv}$ & $289 \pm 77$ \\
\hline VAL 1A & $\mathrm{Fc}$ & $120 \pm 7$ & M.S. 12-6 & $\mathrm{Fv}$ & $292 \pm 44$ \\
\hline LCO 1D & $\mathrm{Fc}$ & $120 \pm 30$ & Gov. Simcoe & $\mathrm{F} \times \mathrm{a}$ & $299 \pm 115$ \\
\hline WSU 88061-3 & $\mathrm{B} \times \mathrm{Fc}$ & $121 \pm 28$ & Senga Sengana & $\mathrm{F} \times \mathrm{a}$ & $308 \pm 110$ \\
\hline VIL 2A & $\mathrm{Fc}$ & $124 \pm 15$ & Sequoia & $\mathrm{F} \times \mathrm{a}$ & $310 \pm 490$ \\
\hline FRA 1178 & Fv & $127 \pm 29$ & M.S. 4-12 & $\mathrm{Fv}$ & $316 \pm 61$ \\
\hline COY 11D & $\mathrm{Fc}$ & $128 \pm 34$ & FRA 552 & $\mathrm{Fv}$ & $316 \pm 44$ \\
\hline ANC 2D & $\mathrm{Fc}$ & $130 \pm 27$ & Glooscap & $\mathrm{F} \times \mathrm{a}$ & $320 \pm 164$ \\
\hline YEN 1P & $\mathrm{Fc}$ & $132 \pm 35$ & FRA 958 & $\mathrm{Fv}$ & $337 \pm 59$ \\
\hline YEN 1J & $\mathrm{Fc}$ & $132 \pm 37$ & M.S. 1-12 & $\mathrm{Fv}$ & $338 \pm 18$ \\
\hline Elsanta & $\mathrm{F} \times \mathrm{a}$ & 137 (1 plant) & Crimson King & $\mathrm{F} \times \mathrm{a}$ & $344 \pm 61$ \\
\hline TDC 1R & $\mathrm{Fc}$ & $140 \pm 23$ & BC 86-33-2 & $\mathrm{F} \times \mathrm{a}$ & $353 \pm 94$ \\
\hline TDC 2B & $\mathrm{Fc}$ & $140 \pm 7$ & $\mathrm{LCO} 3 \mathrm{H}$ & $\mathrm{Fc}$ & $363 \pm 75$ \\
\hline WSU 88061-1 & $\mathrm{B} \times \mathrm{Fc}$ & $140 \pm 48$ & FRA 1179 & $\mathrm{Fv}$ & $364 \pm 73$ \\
\hline FRA 101 & $\mathrm{Fv}$ & $141 \pm 17$ & Honeoye & $\mathrm{F} \times \mathrm{a}$ & $374 \pm 35$ \\
\hline TDC 6B & $\mathrm{Fc}$ & $150 \pm 16$ & White Pine & $\mathrm{F} \times \mathrm{a}$ & $375 \pm 75$ \\
\hline Annapolis & $\mathrm{F} \times \mathrm{a}$ & $162 \pm 34$ & Parker & $\mathrm{F} \times \mathrm{a}$ & $381 \pm 110$ \\
\hline TDC 2D & $\mathrm{Fc}$ & $164 \pm 30$ & CA 71.98-605 & $\mathrm{F} \times \mathrm{a}$ & $387 \pm 82$ \\
\hline TDC 1C & $\mathrm{Fc}$ & $164 \pm 30$ & Scott & $\mathrm{F} \times \mathrm{a}$ & $392 \pm 62$ \\
\hline M.S. 8-24 & Fv & $165 \pm 49$ & M.S. $10-10$ & Fv & $414 \pm 129$ \\
\hline YEN 1B & $\mathrm{Fc}$ & $170 \pm 12$ & Chandler & $\mathrm{F} \times \mathrm{a}$ & 431 (2 plants) \\
\hline
\end{tabular}

virginiana from Minnesota and Wisconsin (Stahler, 1990) were included in this 1993 study. 'Totem', which is susceptible to the mite and aphid, was included as a standard.

Dormant runner plants were planted in a peat-perlite mixture in 2.3-liter plastic pots in Spring 1992 and kept in a greenhouse without supplemental heat or light. Fans kept temperatures near ambient, except if outside temperatures fell below $-4 \mathrm{C}$, heaters kept temperatures at -1 to $0 \mathrm{C}$. Plants were fertilized weekly with $1.3 \mathrm{~g}$ of soluble $20 \mathrm{~N}-20 \mathrm{P}-20 \mathrm{~K}$ fertilizer (Peters Professional Water Soluble Fertilizer; Grace-Sierra, Milpitas, Calif.)/liter of water. Trace amounts of $\mathrm{B}, \mathrm{Cu}, \mathrm{Fe}, \mathrm{Mg}, \mathrm{Mn}, \mathrm{Mo}$, and $\mathrm{Zn}$ also were in the solution. Clones were replicated four times as single plants in a randomized complete-block design.

Spider mite counts typically begin increasing during the flowering period, reach a peak during harvest, and then rapidly decline (Chaplin et al., 1968; Inoue and Sugima, 1984; Marsden, 1974; Poe, 1971; Shanks and Doss, 1989). Therefore, mite counting began at the beginning of bloom and continued every 2 weeks until counts had declined. One leaflet was picked from each of three leaves per plant on each date, and the mites were counted. The six biweekly counts from each replication of each clone were totaled for the period $20 \mathrm{Apr}$. to 29 June 1993.

Resistance to the strawberry aphid first was measured by caging five 7- to 8-day-old nymphs on the underside of one leaflet on each 
Table 1. Continued.

\begin{tabular}{|c|c|c|c|c|c|}
\hline Clone & Species $^{2}$ & $\begin{array}{c}\text { No. mites/three } \\
\text { leaflets } \pm \text { SE }\end{array}$ & Clone & Species $^{\mathrm{z}}$ & $\begin{array}{c}\text { No. mites/three } \\
\text { leaflets } \pm \text { SE }\end{array}$ \\
\hline M.S. 14-23 & $\mathrm{Fv}$ & $171 \pm 40$ & Aiko & $\mathrm{F} \times \mathrm{a}$ & $432 \pm 59$ \\
\hline LON 3D & $\mathrm{Fc}$ & $175 \pm 36$ & Oso Grande & $\mathrm{F} \times \mathrm{a}$ & $453 \pm 73$ \\
\hline COY 10A & $\mathrm{Fc}$ & $184 \pm 11$ & Fern & $\mathrm{F} \times \mathrm{a}$ & $508 \pm 111$ \\
\hline Douglas & $\mathrm{F} \times \mathrm{a}$ & $188 \pm 52$ & Capitola & $\mathrm{F} \times \mathrm{a}$ & $509 \pm 123$ \\
\hline Blomidon & $\mathrm{F} \times \mathrm{a}$ & $189 \pm 59$ & FRA 1171 & $\mathrm{FV}_{\mathrm{V}}$ & $511 \pm 54$ \\
\hline M.S. 33-16 & $\mathrm{FV}$ & $193 \pm 23$ & Seascape & $\mathrm{F} \times \mathrm{a}$ & $552 \pm 172$ \\
\hline M.S. 24-1 & $\mathrm{Fv}$ & $196 \pm 47$ & Muir & $\mathrm{F} \times \mathrm{a}$ & $602 \pm 167$ \\
\hline FRA 1176 & $\mathrm{Fv}$ & $198 \pm 37$ & FRA 1173 & $\mathrm{Fv}_{\mathrm{V}}$ & $619 \pm 73$ \\
\hline FRA 104 & $\mathrm{Fv}_{\mathrm{V}}$ & $202 \pm 11$ & Canoga & $\mathrm{F} \times \mathrm{a}$ & $622 \pm 92$ \\
\hline M.S. $30-15$ & $\mathrm{Fv}$ & $203 \pm 29$ & Selva & $\mathrm{F} \times \mathrm{a}$ & $639 \pm 176$ \\
\hline M.S. 34-4 & Fv & $204 \pm 36$ & Tristar & $\mathrm{F} \times \mathrm{a}$ & $640 \pm 70$ \\
\hline Cardinal & $\mathrm{F} \times \mathrm{a}$ & 207 (3 plants) & FRA 1177 & $\mathrm{FV}_{\mathrm{V}}$ & $643 \pm 146$ \\
\hline Redcrest & $\mathrm{F} \times \mathrm{a}$ & $211 \pm 15$ & CA 69.72-101 & $\mathrm{F} \times \mathrm{a}$ & $658 \pm 121$ \\
\hline Bountiful & $\mathrm{F} \times \mathrm{a}$ & $212 \pm 71$ & Seneca & $\mathrm{F} \times \mathrm{a}$ & $694 \pm 216$ \\
\hline M.S. 27-22 & $\mathrm{Fv}$ & $218 \pm 70$ & Tillikum & $\mathrm{F} \times \mathrm{a}$ & $748 \pm 109$ \\
\hline FRA 994 & $\mathrm{Fv}$ & $223 \pm 16$ & FRA 1181 & $\mathrm{Fv}_{\mathrm{V}}$ & $837 \pm 238$ \\
\hline Shuswap & $\mathrm{F} \times \mathrm{a}$ & $224 \pm 72$ & FRA 1182 & $\mathrm{FV}_{\mathrm{V}}$ & $901 \pm 168$ \\
\hline M.S. 21-5 & $\mathrm{Fv}$ & $226 \pm 47$ & FRA 1174 & $\mathrm{Fv}$ & $946 \pm 178$ \\
\hline
\end{tabular}

${ }^{\mathrm{z}} \mathrm{F} \times \mathrm{a}=$ Fragaria $\times$ ananass $a, \mathrm{Fc}=F$. chiloensis $($ Chile $), \mathrm{Fv}=F$. virginiana $($ eastern North America), $\mathrm{B} \times$ $\mathrm{Fc}=F . \times$ ananassa 'Benton' $\times F$. chiloensis clone CL-5.

of four plants of each clone (Shanks and Garth, 1992). Five days later, the number of survivors (which by then were adults) and nymphs produced were counted. Clones that showed resistance were tested a second time in the same manner, except that the aphids were left on the leaflets for 10 days and the total number of aphids were counted.

Standard errors of the means were calculated for data from screening 171 clones against mites and aphids (Tables 1-3). The data from the longer-term testing against aphids (Table 4) were subjected to analysis of variance for a complete-block design, and means were compared by Tukey's HSD test (Siegel, 1992).

In 1994, two clones of $F$. virginiana from Kentucky, one from Maryland, two from Montana, one from New Hampshire, and one from Washington (all from K. Hummer, NCGR) were tested for resistance to the twospotted spider mite and strawberry aphid as previously described. Mites were counted biweekly from 27 Apr. to 7 July 1994. Also in 1994, 60 clones of $F$. chiloensis that were collected in Chile in 1992 (Cameron et al., 1993) were evaluated. Our collection provided a diverse collection of germplasm from cultivated and wild octaploid Fragaria spp.

\section{Results and Discussion}

Spider mite. Spider mite populations varied from 37.0 to 946 mites per three leaflets in the 1993 trial (Table 1). Eight clones had $\geq 75 \%$ fewer mites than the susceptible standard ('Totem'), and 44 clones had $>50 \%$ fewer than 'Totem'. 'Cavendish' was the only $F$. $\times$ ananassa clone among the eight most resistant clones. WSU 88061-5 and WSU 88061-4 were from a 'Benton' $\times F$. chiloensis 'CL-5' cross. Four were $F$. chiloensis from Chile (Cameron et al., 1991). The large proportion of Chilean $F$. chiloensis that seems to be resistant probably was due to their earlier selection as potentially resistant in an unreplicated trial of $>200$ clones of $F$. chiloensis from Chile (C.H.S., unpublished data). The only $F$. virginiana clone that seemed to be resistant

was FRA 472 (v. platypetala) from Oregon.

In the 1994 trial, 19 Chilean $F$. chiloensis clones had $\geq 90 \%$ fewer mites than did 'Totem' (susceptible), and two (FUT-5A and CPU1A) had $99 \%$ fewer than 'Totem' on 5 July (Table 2). Fifteen clones had fewer mites than F. chiloensis mite-resistant clone CL-5 (C.H.S., unpublished data). Some of the most resistant clones were evaluated for an additional 4 weeks because there was an upsurge in mite counts between 19 June and 5 July on some. On 2 Aug., cumulative mite counts on those clones were still much less than on 'Totem', and mite populations had not increased.

In the 1994 trial, $F$. virginiana clones averaged from $144.0 \pm 51.3$ to $1423 \pm 51$ mites per three leaflets from $28 \mathrm{Apr}$. to 7 July, while 'Totem' averaged $422 \pm 187$ mites per three leaflets (Table 2). Most (77\%) of the mites on FRA 98 occurred on 7 July, so it was not considered to be resistant. Other clones also did not show mite resistance.

Among 21 clones that were included by Giménez-Ferrer et al. (1993) and in our studies (Table 3), those rated intermediate to highly susceptible to twospotted spider mite by the former also were susceptible in our study. 'Totem' was intermediate in relative susceptibility in both studies, but from a practical standpoint, it has been susceptible to spider mites (Barritt and Shanks, 1981; Shanks and Barritt, 1980). Many of the clones ranked intermediate to highly resistant to spider mites by Giménez-Ferrer et al. (1993) were nearly as or more susceptible than 'Totem' in our study. This variation could have been due to environmental differences or a difference in screening method. Giménez-Ferrer et al. (1993) used only one bioassay per clone taken on 1 day during 2 weeks. Shanks and Doss (1989) showed that susceptibility of 'Totem' changed greatly within 2 weeks and cited several other papers that reported similar rapid population declines of spider mites on other strawberry clones.

The level of resistance to spider mites in clones such as 'Annapolis' and 'Cardinal' is certainly preferable to that of 'Totem' and
Table 2. Total number of spider mites on Fragaria spp. clones from 28 Apr. 1994 to the indicated dates.

\begin{tabular}{lccc}
\hline & \multicolumn{3}{c}{ Cumulative no. mites/three leaflets } \\
\cline { 2 - 4 } Clone $^{\mathrm{y}}$ & 5 July & 19 July & 2 Aug. \\
\hline \multicolumn{4}{c}{ F. chiloensis } \\
Totem & $774 \pm 222$ & $822 \pm 253$ & $838 \pm 246$ \\
CL-5 & $45 \pm 15$ & $48 \pm 17$ & $50 \pm 19$ \\
CIS-1A & $1504 \pm 235$ & & \\
FUT-6A & $915 \pm 266$ & & \\
TAP-2A & $538 \pm 195$ & \\
QUI-1A & $523 \pm 219$ &
\end{tabular}

TAP-4C

SIM-1A $\quad 407 \pm 135$

TAP-4A $\quad 402 \pm 128$

CAM-1B $\quad 381 \pm 221$

CAR-1A $\quad 346 \pm 74$

COC-9A $\quad 311 \pm 212$

COC-3A $\quad 288 \pm 80$

MAR-1A $282 \pm 37$

TAP-4B $\quad 279 \pm 79$

CAR-4A $\quad 260 \pm 223$

GRA-1A $\quad 248 \pm 107$

MAR-1B $\quad 245 \pm 135$

CAM-1A $\quad 244 \pm 83$

BER-1A $\quad 232 \pm 128$

TAP-3A $219 \pm 66$

TAP-1A $209 \pm 73$
COC-4A $198 \pm 33$

CAR-3B $\quad 189 \pm 67$

CAM-1C $\quad 171 \pm 140$

BAR-1A $\quad 168 \pm 71$

CUC-1A $\quad 157 \pm 71$

PAL-2B $\quad 156 \pm 59$

$\begin{array}{ll}\text { VAL-1A } & 150 \pm 93\end{array}$

CAR-2A $\quad 149 \pm 32$

$\begin{array}{ll}\text { COC-6A } & 137 \pm 51\end{array}$

$\begin{array}{ll}\text { COC-7A } & 137 \pm 60\end{array}$

BAK-2A $\quad 124 \pm 49$

MAL-1A $122 \pm 58$

MAL-2A $119 \pm 49$

COC-2A $\quad 115 \pm 65$

COC-5A $109 \pm 65$

$\begin{array}{lrr}\text { PAL-2A } & 84 \pm 38 & 169 \pm 53\end{array}$

$\begin{array}{lll}\text { PAL-1A } & 84 \pm 36 & 144 \pm 44\end{array}$

$\begin{array}{llr}\text { COC-1A } & 78 \pm 17 & 80 \pm 17\end{array}$

$\begin{array}{llr}\text { QUI-2A } & 70 \pm 37 & 139 \pm 33\end{array}$

$\begin{array}{lll}\text { BRA-1A } & 67 \pm 45 & 89 \pm 42\end{array}$

GUA-1A $\quad 65 \pm 54 \quad 125 \pm 55$

$\begin{array}{lll}\text { COC-8A } & 59 \pm 23 & 64 \pm 24\end{array}$

FUT-5B $\quad 31 \pm 11 \quad 44 \pm 10$

$\begin{array}{lll}\text { BRA-1B } & 30 \pm 14 & 51 \pm 11\end{array}$

PAL-2C $\quad 26 \pm 18 \quad 58 \pm 23$

YEL-1A $\quad 26 \pm 10 \quad 78 \pm 32$

TOR-1A $\quad 24 \pm 11 \quad 43 \pm 10$

$\begin{array}{lll}\text { CPU-2A } & 24 \pm 9 & 48 \pm 20\end{array}$

$\begin{array}{lll}\text { FUT-4A } & 22 \pm 9 & 102 \pm 19\end{array}$

$\begin{array}{llr}\text { PUQ-1A } & 18 \pm 12 & 78 \pm 31\end{array}$

$\begin{array}{lll}\text { GBN-1A } & 17 \pm 14 & 27 \pm 13\end{array}$

FUT-4B

AMA-2A

$55 \pm 21$

$13 \pm 7 \quad 42 \pm 23$

$11 \pm 4 \quad 42 \pm 14$

$7 \pm 1 \quad 25 \pm 9$

$7 \pm 3 \quad 20 \pm 1$

$64 \pm 24$

$96 \pm 32$

FUT-5A

CPU-1A

F. virginiana ${ }^{w} 7$ July

Totem

CL-5

FRA 98

FRA 434

FRA 381

FRA 67

$422 \pm 187$

$16 \pm 5$

$144 \pm 51$

$193 \pm 91$

$307 \pm 28$

$430 \pm 153$

FRA $560 \quad 577 \pm 72$

FRA $1184 \quad 936 \pm 104$

FRA $1172 \quad 1423 \pm 152$

${ }^{\mathrm{z}}$ Number of mites $\pm \mathrm{SE}$.

'Totem' is mite-susceptible standard; CL-5 is miteresistant $F$. chiloensis standard.

${ }^{x}$ Cameron et al., 1993.

wState of origin is as follows: FRA 98, Montana; FRA 434, Washington; FRA 381, New Hampshire; FRA 67, Maryland; FRA 560, Montana; FRA 1184, Kentucky; FRA 1172, Kentucky.
CHY-1A $\quad 200 \pm 127$

PAL-4A $\quad 24 \pm 20 \quad 44 \pm 17$ 
Breeding, Cultivars, Rootstocks, \& Germplasm Resources

Table 3. Comparison of data from two studies on strawberry resistance to twospotted spider mite.

\begin{tabular}{lccc}
\hline & & \multicolumn{2}{c}{ Current study } \\
\cline { 2 - 4 } Clone & & $\begin{array}{c}\text { Percent } \\
\text { Totem }\end{array}$ & $\begin{array}{c}\text { Percent } \\
\text { Canoga }\end{array}$ \\
\hline Canoga & Ranking $^{\mathrm{y}}$ & 153 & $100^{\mathrm{x}}$ \\
Scott & $\mathrm{S}$ & 96 & 63 \\
Tristar & $\mathrm{I}-\mathrm{S}$ & 157 & 103 \\
Selva & $\mathrm{I}-\mathrm{S}$ & 157 & 103 \\
Muir & $\mathrm{I}-\mathrm{S}$ & 148 & 97 \\
Oso Grande & $\mathrm{I}-\mathrm{S}$ & 111 & 73 \\
Honeoye & $\mathrm{I}-\mathrm{S}$ & 92 & 60 \\
Crimson King & $\mathrm{I}-\mathrm{S}$ & 84 & 55 \\
Totem & $\mathrm{I}-\mathrm{S}$ & $100^{\mathrm{w}}$ & 66 \\
Blomidon & $\mathrm{I}-\mathrm{R}$ & 46 & 30 \\
Earliglow & $\mathrm{I}-\mathrm{R}$ & 66 & 43 \\
Chandler & $\mathrm{I}-\mathrm{R}$ & 90 & 59 \\
Douglas & $\mathrm{R}$ & 46 & 30 \\
Govenor Simcoe & $\mathrm{R}$ & 73 & 48 \\
Glooscap & $\mathrm{R}$ & 79 & 51 \\
Parker & $\mathrm{R}$ & 94 & 61 \\
Annapolis & $\mathrm{HR}$ & 40 & 26 \\
Cardinal & $\mathrm{HR}$ & 51 & 33 \\
Pajaro & $\mathrm{HR}$ & 69 & 45 \\
Aiko & $\mathrm{HR}$ & 106 & 69 \\
Fern & $\mathrm{HR}$ & 124 & 82 \\
\hline Rank according & & & \\
\hline
\end{tabular}

${ }^{2}$ Rank according to oviposition on leaf disks (Giménez-Ferrer et al., 1993).

${ }^{y} \mathrm{HS}=$ highly susceptible, $\mathrm{S}=$ susceptible, $\mathrm{I}-\mathrm{S}=$ intermediate to susceptible, $\mathrm{I}-\mathrm{R}=$ intermediate to resistant, $\mathrm{R}=$ resistant, $\mathrm{HR}=$ highly resistant.

${ }^{x}$ 'Canoga' was the most susceptible clone in this study and had a total of 622 mites per leaflet.

w'Totem' was the mite-susceptible clone in our study and had a total of 408 mites per leaflet.

other even more susceptible clones. However, there is a large amount of mite-resistant germplasm available, which should make it possible to develop even higher levels of resistance to spider mites in strawberries. The clones 'Cavendish', WSU 88061-4, and WSU 880615 are examples of $F$. xananassa with lower susceptibility to spider mites. Also, several $F$. chiloensis and $F$. virginiana clones had high levels of mite resistance compared to other resistant clones. This pool of germplasm should be useful to strawberry breeders in developing spider-mite-resistant cultivars, which would reduce or eliminate the need for chemical acaricides.

Aphids. PNN 6A, M.S. 30-15, M.S. 6-4, and 'Scott' were the only clones of the three Fragaria species showing any evidence for aphid resistance in the 5-day test (Table 4). Aphids had high survival and reproductive rates on most of the clones tested. One $F$. chiloensis, three $F$. virginiana, and six $F$. Xananassa were selected for the 10-day test because they averaged less than two survivors per leaflet in the 5-day test. WSU 2068 also was included because few nymphs were produced in the 5-day test. In the 10-day test, M.S. 6-4, M.S. 30-15, 'Scott', and 'Elsanta' had $>90 \%$ fewer aphids than did 'Totem' after 10 days (Table 5). PNN 6A was the only clone to show resistance to spider mites and the strawberry aphid and was only moderately resistant to aphids in the 10-day aphid test.

Aphid-borne viruses are a problem to the strawberry industry, although most currently grown cultivars have some tolerance to viruses bred into them. Resistance to the straw-
Table 4. Five-day survival of strawberry aphids on various clones of Fragaria

\begin{tabular}{|c|c|c|c|c|c|}
\hline Clones & $\begin{array}{c}\text { Mean no. } \\
\text { survivors } \\
( \pm S E)^{2}\end{array}$ & $\begin{array}{c}\text { Mean no. } \\
\text { nymphs } \\
( \pm \mathrm{SE})\end{array}$ & Clone & $\begin{array}{c}\text { Mean no. } \\
\text { survivors } \\
( \pm \mathrm{SE})\end{array}$ & $\begin{array}{c}\text { Mean no } \\
\text { nymphs } \\
( \pm \mathrm{SE})\end{array}$ \\
\hline \multicolumn{6}{|c|}{ Expt. 1-Fragaria chiloensis ${ }^{y}$} \\
\hline Totem $^{x}$ & $4.8 \pm 0.25$ & $13.0 \pm 1.6$ & TDC 1R & $4.5 \pm 0.50$ & $11.0 \pm 4.6$ \\
\hline PNN 6A & $0.8 \pm 0.75$ & $4.0 \pm 4.0$ & YEN 1H & $4.5 \pm 0.50$ & $10.0 \pm 4.3$ \\
\hline LCO 1D & $3.3 \pm 1.03$ & $8.8 \pm 4.7$ & ANC 2D & $4.8 \pm 0.25$ & $10.5 \pm 1.6$ \\
\hline YEN 1B & $3.3 \pm 0.85$ & $13.5 \pm 4.0$ & $\mathrm{LCO} 1 \mathrm{C}$ & $4.8 \pm 0.25$ & $15.0 \pm 5.5$ \\
\hline WSU 88061-2 & $3.5 \pm 1.19$ & $12.8 \pm 5.8$ & $\mathrm{LCO} 3 \mathrm{H}$ & $4.8 \pm 0.25$ & $21.3 \pm 2.8$ \\
\hline WSU 88061-5 & $3.5 \pm 0.65$ & $9.5 \pm 4.7$ & LON 3D & $4.8 \pm 0.25$ & $12.3 \pm 3.2$ \\
\hline TDT 5B & $3.5 \pm 0.87$ & $9.8 \pm 2.1$ & PUR 1A-2 & $4.8 \pm 0.25$ & $14.0 \pm 4.9$ \\
\hline TDC 2D & $3.8 \pm 0.95$ & $6.3 \pm 2.9$ & TDC 1C & $4.8 \pm 0.25$ & $20.3 \pm 3.2$ \\
\hline WSU 88061-6 & $3.8 \pm 0.95$ & $6.5 \pm 3.2$ & TDC 2B & $4.8 \pm 0.30$ & $18.5 \pm 2.2$ \\
\hline YEN 1Q & $3.8 \pm 0.75$ & $9.5 \pm 3.9$ & YEN 1I & $4.8 \pm 0.25$ & $17.5 \pm 1.8$ \\
\hline VAL 1A & $4.0 \pm 0.41$ & $8.3 \pm 3.5$ & YEN 1J & $4.8 \pm 0.25$ & $18.8 \pm 4.5$ \\
\hline WSU 88061-4 & $4.3 \pm 0.25$ & $6.5 \pm 2.3$ & YEN 1P & $4.8 \pm 0.25$ & $18.8 \pm 4.4$ \\
\hline BAM 1E & $4.3 \pm 0.25$ & $5.3 \pm 2.2$ & WSU 88061-3 & $5.0 \pm 0$ & $14.0 \pm 1.7$ \\
\hline COY 11D & $4.3 \pm 0.25$ & $11.3 \pm 3.1$ & COY $10 \mathrm{~A}$ & $5.0 \pm 0$ & $19.3 \pm 4.7$ \\
\hline TDC 6B & $4.3 \pm 0.48$ & $9.0 \pm 3.7$ & TDT 1D & $5.0 \pm 0$ & $12.0 \pm 6.3$ \\
\hline WSU 88061-1 & $4.5 \pm 0.29$ & $9.3 \pm 3.8$ & VIL 2A & $5.0 \pm 0$ & $18.5 \pm 2.4$ \\
\hline MAU 1C & $4.5 \pm 0.50$ & $10.8 \pm 5.1$ & & & \\
\hline \multicolumn{6}{|c|}{ Expt. 2-Fragaria virginiana ${ }^{y}$} \\
\hline Totem & $4.8 \pm 0.25$ & $11.3 \pm 2.6$ & M.S. 34-4 & $4.3 \pm 0.25$ & $8.5 \pm 2.7$ \\
\hline M.S. 30-15 & $0.3 \pm 0.25$ & $0.0 \pm 0$ & FRA 1170 & $4.5 \pm 0.29$ & $3.5 \pm 2.4$ \\
\hline M.S. 6-4 & $0.5 \pm 0.29$ & $1.3 \pm 1.3$ & FRA 1178 & $4.5 \pm 0.29$ & $8.8 \pm 4.3$ \\
\hline FRA 1181 & $1.8 \pm 0.75$ & $3.3 \pm 2.9$ & FRA 1180 & $4.5 \pm 0.29$ & $6.8 \pm 4.3$ \\
\hline M.S. $4-12$ & $2.8 \pm 0.48$ & $4.3 \pm 2.3$ & FRA 960 & $4.5 \pm 0.29$ & $11.5 \pm 5.6$ \\
\hline FRA 1171 & $3.0 \pm 0.71$ & $6.0 \pm 3.7$ & M.S. 1-12 & $4.5 \pm 0.50$ & $17.8 \pm 6.8$ \\
\hline FRA 958 & $3.5 \pm 0.87$ & $9.0 \pm 3.2$ & M.S. 33-16 & $4.5 \pm 0.29$ & $6.8 \pm 3.1$ \\
\hline FRA 552 & $3.8 \pm 0.95$ & $5.5 \pm 2.2$ & FRA 101 & $4.8 \pm 0.25$ & $13.8 \pm 2.0$ \\
\hline FRA 994 & $3.8 \pm 0.95$ & $13.3 \pm 5.7$ & FRA 1174 & $4.8 \pm 0.25$ & $4.3 \pm 3.9$ \\
\hline M.S. $27-22$ & $3.8 \pm 1.25$ & $10.0 \pm 4.0$ & FRA 1179 & $4.8 \pm 0.25$ & $13.3 \pm 1.9$ \\
\hline M.S. $10-10$ & $4.0 \pm 1.00$ & $14.5 \pm 7.8$ & FRA 1182 & $4.8 \pm 0.25$ & $9.5 \pm 3.4$ \\
\hline M.S. $14-23$ & $4.0 \pm 0.41$ & $7.5 \pm 0.6$ & FRA 472 & $4.8 \pm 0.25$ & $14.8 \pm 3.8$ \\
\hline M.S. 24-1 & $4.0 \pm 0.71$ & $4.5 \pm 2.6$ & FRA 993 & $4.8 \pm 0.25$ & $12.5 \pm 2.2$ \\
\hline FRA 1007 & $4.3 \pm 0.75$ & $9.0 \pm 3.5$ & M.S. $12-6$ & $4.8 \pm 0.25$ & $10.5 \pm 4.7$ \\
\hline FRA 1173 & $4.3 \pm 0.75$ & $8.3 \pm 1.7$ & FRA 104 & $5.0 \pm 0$ & $16.8 \pm 4.4$ \\
\hline FRA 1177 & $4.3 \pm 0.75$ & $14.8 \pm 1.0$ & FRA 1176 & $5.0 \pm 0$ & $10.3 \pm 1.7$ \\
\hline M.S. 21-5 & $4.3 \pm 0.75$ & $7.3 \pm 1.8$ & M.S. 8-24 & $5.0 \pm 0$ & $8.3 \pm 2.3$ \\
\hline \multicolumn{6}{|c|}{ Expt. 3-Fragaria $\times$ ananassa $^{y}$} \\
\hline Totem & $4.0 \pm 0.58$ & $6.3 \pm 2.5$ & Douglas & $3.3 \pm 0.48$ & $5.5 \pm 2.1$ \\
\hline Scott & $0.5 \pm 0.29$ & $0.3 \pm 0.3$ & Muir & $3.3 \pm 1.03$ & $6.0 \pm 3.6$ \\
\hline Canoga & $1.0 \pm 0.71$ & $3.8 \pm 1.9$ & Oso Grande & $3.3 \pm 1.11$ & $3.3 \pm 2.0$ \\
\hline Gov. Simcoe & $1.0 \pm 0.41$ & $5.0 \pm 4.1$ & Tillikum & $3.3 \pm 1.18$ & $11.8 \pm 6.6$ \\
\hline Elsanta & $1.3 \pm 0.75$ & $0.5 \pm 0.3$ & CA. $71.98-605$ & $3.3 \pm 0.75$ & $8.5 \pm 5.1$ \\
\hline CA. $69.72-101$ & $1.5 \pm 1.19$ & $1.3 \pm 0.8$ & Cardinal & $3.5 \pm 0.50$ & $4.8 \pm 1.4$ \\
\hline Capitola & $1.8 \pm 1.18$ & $1.8 \pm 1.0$ & Earliglow & $3.5 \pm 1.19$ & $1.5 \pm 1.0$ \\
\hline Seascape & $2.0 \pm 0.91$ & $3.0 \pm 2.4$ & Senga Sengana & $3.5 \pm 1.19$ & $4.0 \pm 2.5$ \\
\hline Seneca & $2.0 \pm 1.00$ & $3.3 \pm 2.9$ & Aiko & $3.8 \pm 1.25$ & $1.5 \pm 0.9$ \\
\hline $342-\mathrm{A}-65$ & $2.0 \pm 1.15$ & $11.3 \pm 3.8$ & Blomidon & $4.0 \pm 0.71$ & $8.0 \pm 4.1$ \\
\hline WSU 2068 & $2.0 \pm 1.08$ & $0.8 \pm 0.8$ & Cavendish & $4.0 \pm 0.71$ & $8.3 \pm 3.4$ \\
\hline Gorella & $2.3 \pm 1.11$ & $3.8 \pm 2.3$ & Honeoye & $4.0 \pm 0.58$ & $8.8 \pm 2.9$ \\
\hline Parker & $2.3 \pm 0.95$ & $2.0 \pm 0.8$ & Sequoia & $4.0 \pm 1.00$ & $8.0 \pm 2.4$ \\
\hline Fern & $2.5 \pm 0.87$ & $3.3 \pm 1.9$ & ВC 86-33-2 & $4.0 \pm 0.71$ & $12.3 \pm 3.6$ \\
\hline Shuswap & $2.5 \pm 0.50$ & $6.0 \pm 2.3$ & Glooscap & $4.3 \pm 0.48$ & $12.8 \pm 6.5$ \\
\hline Pajaro & $2.8 \pm 1.31$ & $4.8 \pm 2.5$ & Redcrest & $4.3 \pm 2.5$ & $9.8 \pm 2.5$ \\
\hline Selva & $2.8 \pm 1.11$ & $11.3 \pm 9.4$ & Tristar & $4.5 \pm 0.5$ & $8.3 \pm 3.6$ \\
\hline White Pine & $3.0 \pm 0.82$ & $1.8 \pm 0.5$ & Annapolis & $4.8 \pm 0.25$ & $10.8 \pm 2.0$ \\
\hline Chandler & $3.3 \pm 0.85$ & $4.5 \pm 3.8$ & Bountiful & $5.0 \pm 0$ & $21.0 \pm 2.4$ \\
\hline Crimson King & $3.3 \pm 1.18$ & $1.5 \pm 0.9$ & & & \\
\hline
\end{tabular}

${ }^{2}$ Five aphids per replicate.

${ }^{y}$ See Table 1 for source of each clone.

${ }^{\mathrm{x}}$ Susceptible standard.

berry aphid, the principal vector of strawberry viruses, would be an additional defense against these pathogens. Swenson (1968) stated that any factor that consistently reduces aphid populations can be expected to reduce virus spread. This is the principle behind an area-wide spray program for reducing strawberry aphid populations and virus dissemination in the Pacific Northwest (Shanks, 1986). Using aphid-resistant cultivars would reduce or eliminate the need for the aphicide sprays.

\section{Literature Cited}

Barritt, B.H. and C.H. Shanks, Jr. 1981. Parent selection in breeding strawberries resistant to twospotted spider mites. HortScience 16:323324.

Cameron, J.S., C.H. Shanks, Jr., T.M. Sjulin, and C.E. Munoz. 1991. Collection of Fragaria germplasm from central and southern Chile, p. 108110. In: A. Dale and J. Luby (eds.). The strawberry into the 21 st century. Timber Press, Portland, Ore. 
Table 5. Total number of adult and immature strawberry aphids on strawberry clones after 10 days.

\begin{tabular}{lc}
\hline \hline & $\begin{array}{c}\text { Mean no. } \\
\text { aphids/replicate } \\
\text { Clone }^{\mathrm{z}}\end{array}$ \\
\hline M.S. 30-15 SE $)^{\mathrm{y}, \mathrm{x}}$
\end{tabular}

${ }^{2}$ See Table 1 for source of Fragaria chiloensis and $F$. virginiana clones.

y Four replicates.

${ }^{x}$ Data transformed to $\log (X+1)$ before analysis and transformed to original scale for presentation. Numbers followed by the same letter are not significantly different at $P \leq 0.05$ using Tukey's HSD test.

${ }^{\text {w}}$ Aphid-susceptible standard.
Cameron, J.S., T.M. Sjulin, J.R. Ballington, C.H. Shanks, C.E. Munoz, and A. Lavin. 1993. Exploration, collection and evaluation of Chilean Fragaria: Summary of 1990 and 1992 expeditions. Acta Hort. 348:65-74.

Chaplin, C.E., L.P. Stoltz, and J.G. Rodriguez. 1968. The inheritance of resistance to the two-spotted mite Tetranychus urticae Koch in strawberries. Proc. Amer. Soc. Hort. Sci. 92:376-380.

Giménez-Ferrer, R.M.G., J.C. Scheerens, and W.A. Erb. 1993. In vitro screening of 76 strawberry cultivars for twospotted spider mite resistance. HortScience 28:841-844.

Hancock, J.F., J.L. Maas, C.H. Shanks, P.J. Breen, and J.J. Luby. 1991. Strawberries (Fragaria), p. 491-546. In: J.N. Moore and J.R. Ballington (eds.). Genetic resources of temperate fruit and nut crops. Intl. Soc. Hort. Sci., Wageningen, The Netherlands.

Inoue, M. and T. Sugiura. 1984. Studies of twospotted spider mite Tetranychus urticae Koch on vegetable plants (II). Increasing patterns of mite population in the vinyl house of forced 'Hokowase' strawberries and possibility of its control with mitecide [sic] application on infested areas. Bul. Nara Agr. Expt. Sta. 15:59-65.

Marsden, D.A. 1974. The effects of host plant resistance on the population dynamics of the twospotted spider mite, (Tetranychus
urticae:Koch), on strawberry. PhD Diss., Univ. of California, Berkeley.

Poe, S.L. 1971. Influence of host plant physiology on populations of Tetranychus urticae (Acarina: Tetranychidae) infesting strawberry plants in peninsular Florida. Fla. Entomol. 54:183-186.

Shanks, C. 1986. Strawberry aphids and strawberry viruses. Washington State Univ. Coop. Ext. Bul. 1012.

Shanks, C.H., Jr., and B.H. Barritt. 1980. Twospotted spider mite resistance of Washington strawberries. J. Econ. Entomol. 73:419-423.

Shanks, C.H., Jr., and R.P. Doss. 1989. Population fluctuations of twospotted spider mite (Acari:Tetranychidae) on strawberry. Environ. Entomol.18:641-645.

Shanks, C.H., Jr., and J.K.L. Garth. 1992. Honeydew production, survival and reproduction by Chaetosiphon fragaefolii (Cockerell) (Homoptera:Aphididae) on susceptible and resistant clones of Fragaria spp. Sci. Hort. 50:71-77.

Siegel, J. (ed.). 1992. Statistix, version 4. Analytical Software, St. Paul, Minn.

Stahler, M.M. 1990. Evaluation of variation in Minnesota/Wisconsin Fragaria virginiana for horticultural and morphological traits. PhD Diss. Univ. of Minnesota, Minneapolis St. Paul.

Swenson, K.G. 1968. Role of aphids in the ecology of plant viruses. Annu. Rev. Phytopathol. 6:351-374. 